\title{
Random Sampling of the Protein Data Bank - RaSPDB
}

Oliviero Carugo ( $\boldsymbol{\nabla}$ Oliviero.carugo@univie.ac.at)

University of Pavia

\section{Research Article}

Keywords: Protein Data Bank, Random numbers, Redundancy, Sampling, Standard error

Posted Date: October 7th, 2021

DOI: https://doi.org/10.21203/rs.3.rs-952385/v1

License: (a) (1) This work is licensed under a Creative Commons Attribution 4.0 International License. Read Full License

Version of Record: A version of this preprint was published at Scientific Reports on December 1st, 2021. See the published version at https://doi.org/10.1038/s41598-021-03615-y. 


\section{Abstract}

A novel and simple procedure (RaSPDB) for Protein Data Bank mining is described. 10 PDB subsets, each containing 7000 randomly selected protein chains, are built and used to make 10 estimations of the average value of a generic feature $F$ - the length of the protein chain, the amino acid composition, the crystallographic resolution, and the secondary structure composition. These 10 estimations are then used to compute an average estimation of $\mathrm{F}$ together with its standard error. It is heuristically verified that the dimension of these 10 subsets -7000 protein chains - is sufficiently small to avoid redundancy within each subset and sufficiently large to guarantee stable estimations amongst different subsets. RaSPDB has two major advantages over classical procedures aimed to build a single, non-redundant PDB subset: a larger fraction of the information stored in the PDB is used and an estimation of the standard error of $F$ is possible.

\section{Introduction}

The Protein Data Bank (PDB) is the largest collection of macromolecule three-dimensional structures determined experimentally with X-ray crystallography, cryo-EM, NMR, and with other, relatively uncommon techniques [1][2]. It has countless applications: it is in the PDB that crystallographers find the information necessary to solve the phase problem with molecular replacement methods [3]; bioinformaticians find there templates for homology modelling of protein structures [4]; drug discovery has been greatly influenced by PDB too [5]; and the information deposited in the PDB has been widely used to delineate structural trends and prediction tools, dealing with secondary structure [6], protein motifs [7] or accessibility to internal, buried cavities [8], just to mention few examples.

For estimating any feature $\Phi$ from the PDB it is necessary to build a non-redundant subset of the PDB [9]. This is generally performed by selecting an arbitrary maximum percentage of sequence identity (PMAX), usually in the $20-40 \%$ range, so that the resulting subset contains only entries with percentages of sequence identity lower than PMAX [10][11]. This is necessary, since the PDB is known to be rather redundant [12] and, as a consequence, only biased estimations of any $\Phi$ can be determined from it.

Although the redundancy limitation is a well-accepted procedure, it has two main drawbacks; it reduces, sometime enormously, the amount of information stored in the PDB and it does not allow the estimation of the standard error of $\Phi$.

An alternative procedure, resembling a blocking factor analysis [13] and referred to as RaSPDB, is described in the present communication: Nequally populated subsets of the PDB, each containing $D$ protein chains, are randomly assembled, as in blocking factor analysis.

An evident advantage of $R A S P D B$ is that the value of $\Phi$ can be computed $N$ times, each time being independent of the others, and that it is consequently possible to compute the average value of the $N$ values of $\Phi$ together with its standard error and, if needed, other figures of merit related to the distribution 
of the $N$ values of $\Phi$. This is impossible by following the traditional procedure, where a single subset of the PDB is built and used as a unique source of information.

Moreover, this RaSPDB allows the use of a larger amount of the information stored in the PDB.

A possible problem of $R a S P D B$ is the redundancy level within the $N$ subsets containing $D$ files. However, if $D$ is sufficiently small relative to the dimension of the PDB, this is clearly a minor problem, since the probability to pick-up homologous entries is very small. On the contrary, if $D$ is large, it would be not surprising to find homologous entries in the same subset.

Apropos of redundancy, small $D$ values - which means small subsets of the PDB - are thus advisable. However, if $D$ is too small it is possible that the estimations of $\Phi$ are extremely unstable from one subset to the next. In other words, it is impossible to obtain reliable estimations of $\Phi$ if sampling is insufficient. For this reason, the dimension of the subsets cannot be too small.

It is consequently necessary to attain a compromise solution, in which $D$ must be small enough to avoid excessive redundancy and big enough to ensure sufficient sampling.

Worthy to be remembered, eventually, that RaSPDB cannot substitute additional criteria for assembling high quality subsets of the PDB, like R-factor, free-R-factor, crystallographic resolution and others [9]. It can, however, flank these strategies for improving PDB mining.

\section{Results And Discussion Random Sampling}

In the present communication, the number of subsets is fixed to $N=10$ and the dimension of each subset is varied from 100 to $10,000(D=100,500,1000,2000,3000,4000,5000,6000,7000,8000,9000$ or $10,000)$. The random assembly of the subset was performed as described in the Supplementary Material.

\section{F stability}

A series of features $F$ were examined, some related to sequence (the length of the protein chain defined by its number of residues, and the amino acid composition) and others related to structure (the crystallographic resolution and the secondary structure composition).

Figure 1 shows how the length of the protein chain depends on $D$. As expected, if $D$ is small (for example $D=100$ ), the average protein length is quite variable amongst the $N=10$ subsets. It ranges from 226.5 to 267.9 with an average value of 245.1 and an estimated standard error of 4.0. On the contrary, if $D$ is larger (for example $D=10000$ ), the average protein length is much less variable amongst the $N=10$ subsets. The difference between the maximal (250.3) and minimal (244.9) values is much smaller and the average value (247.8) is associated with a much smaller estimated standard error (0.5). 
The difference between the maximal and minimal value of protein length decreases when $D$ increases, up to $D=6000$. It goes from 41.4 to $27.2,22.1,10.6,7.9,7.1,7.7$ and 6.4 when $D$ goes from 100 to 500,1000 , $2000,3000,4000,5000$ and 6000 . Then is close to 5 and nearly constant for larger $D$ values (4.9 for $D=$ $7000,5.0$ for $D=8000,4.9$ for $D=9000$ and 5.4 for $D=10000$ ).

The estimated standard errors decrease too when $D$ increases, going from 4.0 for $D=100$ to 0.74 for $D=$ 6000. Then, for larger $D$ values, they are nearly constant $(0.57$ for $D=7000,0.61$ for $D=8000,0.52$ for $D=$ 9000 and 0.52 for $D=10000$ ).

Note that both protein length average values and standard errors are computed on the ten average values, each of which is computed with one of the ten subsets of size $D$. The decrease of the standard errors is thus not due to the growing of the number of data used to computed them.

This trend is observed not only when $\mathrm{F}$ is the length of the protein chain. Figures S1-S3 (Supplementary material) show the dependence on $D$ of other features, the crystallographic resolution, the secondary structure composition, and the amino acid composition.

By visual inspection it is possible to estimate that, on average, the divergence between the estimations of $\mathrm{F}$ in different subsets of the PDB decreases up the $D=6000$ to become stable for larger subsets with $D$ $\geq 7000$.

It is thus reasonable to deduce that subsets of the PDB containing 7000 protein chains are large enough for sampling the corpus of protein structures deposited in the PDB.

\section{Internal redundancy}

It is now necessary to check the level of redundancy in randomly selected subsets of the PDB that contain 7000 protein chains.

Given that an all-against-all sequence alignments would have been too expensive - nearly 25 million alignments for each of the ten PDB subsets - only 10000 randomly selected alignments were considered for each subset.

The average redundancy is very small. Amongst the ten PDB subsets: the average pairwise percentage of sequence identity ranges from $9.74 \%$ to $11.18 \%$, with a mean value of $10.52 \%$ (estimated error $0.06 \%$ ).

The percentage of sequences pairs with percentage of sequence identity larger than $40 \%$ is also small $(0.06 \%)$.

This clearly shows that randomly built PDB subsets containing 7000 protein chains are sufficiently small to avoid excessive internal redundancy. 
Furthermore, it is necessary to check how frequently the same protein sequence is randomly selected in two or more of the 10 PDB subsets. In other words, it is necessary to evaluate the degree of overlap between the subsets. This is a relevant problem, since there are 70000 entries in ten groups of 7000 entries and this represents a considerable fraction (about 40\%) of the whole PDB.

The overlap between the two groups, each containing $D$ protein sequences, is evaluated by removing duplicate sequences from one of them. As a consequence, two groups might contain $R$ and $R^{\prime}$ protein sequences, with $R \leq D$ and $R^{\prime} \leq D$. The expression NOVP $=100 \min (R, R) / D$ would be equal to 100 if no overlap occurs, and it would be lower than 100 in case of overlap. Here NOVP ranges from 66 to 100, amongst all unique pairs of the ten PDB random subsets, with an average vale of 81 (standard error 3 ). This indicates that it is uncommon that the same structure is selected twice in ten PDB subsets of 7000 protein chains.

\section{Methods}

A file containing sequences in FASTA format for all entries in the PDB archive was downloaded from http://www.rcsb.org/downloads/fasta. After removing all non-protein sequences, $\mathrm{N}$ groups containing $\mathrm{D}$ protein chains - with $N=10$ and $D=100,500,1000,2000,3000,4000,5000,6000,7000,8000,9000$ or 10000 - were randomly extracted (see Supplementary Material).

Secondary structures were assigned with STRIDE [14], lengths of protein chains were determined from the SEQRES lines of the PDB files. Sequence alignments were performed with the Needleman-Wunsch algorithm, coded into the EMBOSS routine Needle [15]. All statistical analyses were performed with locally written software.

\section{Declarations}

\section{Acknowledgements}

B. Marcello is gratefully acknowledged for helpful discussion and constant support and K. DjinovićCarugo is acknowledged for her kind hospitality in Vienna.

\section{Author contributions}

OC designed the project, performed the computations, and wrote the manuscript.

\section{Additional information}

The author declares no competing interests.

\section{References}

1. Protein Data Bank. Crystallography: Protein Data Bank. Nat New Biol. 1971;233. 
2. wwPDB Consortium. Protein Data Bank: The single global archive fro 3D macromolecular structural data. Nucleic Acids Res. 2019;47:D520-8.

3. Drenth J. Principles of Protein X-ray Crystallography. New York: Springer-Verlag Inc.; 1994.

4. Tramontano A. Protein Structure Prediction: Concepts and Applications. New York: John Wiley \& Sons; 2006.

5. Burley SK. Impact of structural biologists and the Protein Data Bank on small-molecule drug discovery and development. J Biol Chem. 2021;296:100559.

6. Pirovano W, Heringa J. Protein secondary structure prediction. Methods Mol Biol. 2010;609:327-48.

7. Thornton JM, Gardner SP. Protein motifs and data-base searching. Trends Biochem Sci. 1989;14:3004.

8. Carugo O, Argos P. Accessibility to internal cavities and ligand binding sites monitored by protein crystallographic thermal factors. Proteins. 1998;31:201-13.

9. Djinovic-Carugo K, Carugo O. Criteria to extract high quality Protein Data Bank subsets for structure users. Methods Mol Biol. 2016;1415:139-52.

10. Griep S, Hobohm U. PDBselect 1992-2009 and PDBfilter-select. Nucleic Acids Res. 2010;38:D318-319.

11. Fu L, Niu B, Zhu Z, Wu S, Li W. CD-HIT: accelerated for clustering the next generation sequencing data. . Bioinformatics. 2012;28:3150-2.

12. Gromiha MM. Protein Bioinformatics: from Sequence to Function. Elsevier; 2010.

13. Wit E, McClure J. Statistics for Microarrays: Design, Analysis and Inference. John Wiley \& Sons; 2004.

14. Heinig M, Frishman D. STRIDE: A web server for secondary structure assignment from known atomic coordinates of proteins. Nucleic Acids Res. 2004;32:w500-2.

15. Rice P, Longden I, Bleasby A. EMBOSS: the European Molecular Biology Open Software Suite. Trends Genet. 2000;16:276-7.

\section{Figures}

\section{Figure 1}

Relationship between protein chain length and dimension of the randomly selected subset of the PDB. 


\section{Supplementary Files}

This is a list of supplementary files associated with this preprint. Click to download.

- RaSPDBsupplementary.docx 